\title{
Designing Dispersion- and Mode-Area-Decreasing Holey Fibers for Soliton Compression
}

\author{
M. L. V. Tse, P. Horak, F. Poletti, and D. J. Richardson \\ Optoelectronics Research Centre, University of Southampton, Southampton, SO17 1BJ, UK \\ mlt@orc.soton.ac.uk
}

\begin{abstract}
We investigate numerically the adiabatic compression of solitons at $1.55 \mu \mathrm{m}$ in holey fibers which exhibit simultaneously decreasing dispersion and effective mode area. Compression factors $>10$ are achieved for optimum fiber parameters.

(C) 2007 Optical Society of America

OCIS codes: (060.4370) Nonlinear optics, fibers; (230.3990) Microstructure devices; (320.5520) Pulse compression.
\end{abstract}

\section{Introduction}

Compression of soliton pulses propagating in optical fibers with decreasing dispersion is a well-established technique [1]. Very recently, a generalization of the technique for picosecond pulses has been suggested using tapered holey fibers [2]. This has been successfully demonstrated with femtosecond pulses at $1.06 \mu \mathrm{m}$ [3].

Here, we investigate in more detail the idea of using dispersion- and mode-area-decreasing silica holey fibers and we analyze the required fiber design parameters. As a starting point, we show in Fig. 1(a) the dependence of dispersion $D$, dispersion slope Ds, and effective mode area $A_{\text {eff }}$ on pitch $\Lambda$ and air-filling fraction $d / \Lambda$ of holey fibers with regular hexagonal geometry at $1.55 \mu \mathrm{m}$ wavelength. We note that by appropriate fiber fabrication and/or tapering, a large variation of both $\mathrm{D}$ and $\mathrm{A}_{\mathrm{eff}}$ can be achieved along the length of a single fiber.

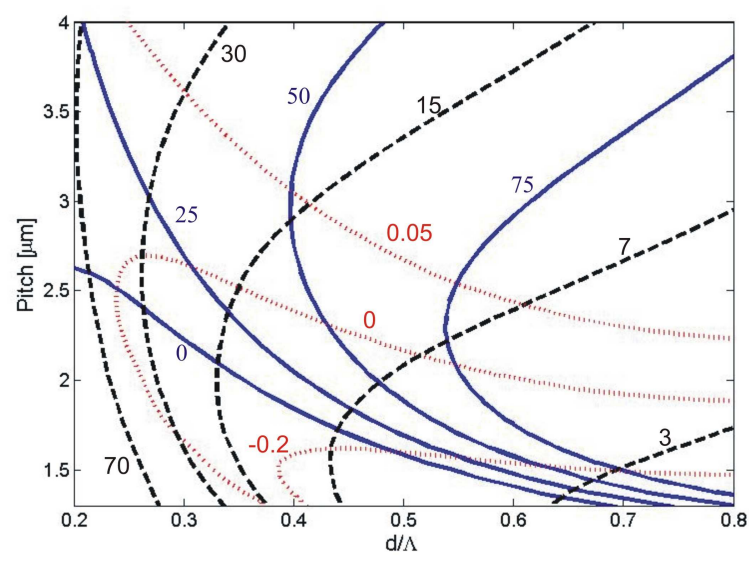

(a)

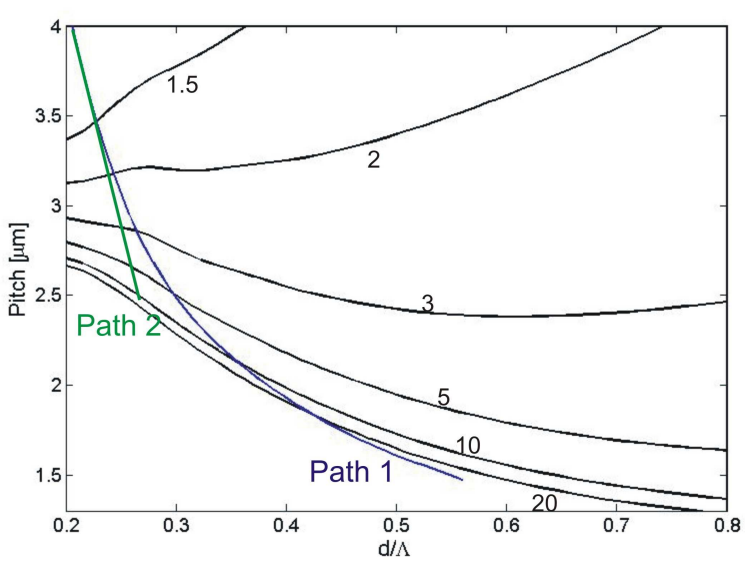

(b)

Fig. 1 Contour maps for (a) dispersion (blue solid) $(\mathrm{ps} / \mathrm{nm} / \mathrm{km})$, dispersion slope (red dotted) $\left(\mathrm{ps} / \mathrm{nm}^{2} / \mathrm{km}\right)$ and effective area (black dashed) $\left(\mu \mathrm{m}^{2}\right)$, (b) adiabatic compression factors versus pitch $\Lambda$ and $\mathrm{d} / \Lambda$ for holey fibers of hexagonal geometry at $1.55 \mu \mathrm{m}$ wavelength.

\section{Theory}

For given fiber parameters and pulse energy, the width of a fundamental soliton is

$$
\tau_{0}=\frac{\lambda^{3} D A_{e f f}}{2 \pi^{2} c n_{2} E_{\text {sol }}},
$$

where $\lambda$ is the wavelength, $n_{2}$ is the nonlinear-index coefficient, and $E_{\text {sol }}$ is the soliton energy. For adiabatic soliton compression in a low-loss fiber, $E_{\text {sol }}$ stays approximately constant and the pulse width is proportional to the product of dispersion coefficient and effective area. Based on equation (1), we thus obtain the adiabatic compression factor, Fig. 1(b), corresponding to the map of fiber parameters, Fig. 1(a). Note that Fig. 1(b) is normalized to the top left corner of the figure which has the largest value of $\mathrm{D}^{*} \mathrm{~A}_{\mathrm{eff}}$. A tapered fiber with parameters changing from that point to any other point on the map will result in compression of a soliton at $1.55 \mu \mathrm{m}$ by the factor shown in the figure, 
provided that changes of fiber parameters over one local dispersion length are small. We performed numerical simulations of the generalized nonlinear Schrödinger equation using a standard split-step Fourier tool for two fibers with parameters following paths 1 and 2, respectively, as shown in Fig. 1(b).

\section{Simulations and Results}

For Path 1 from Fig. 1(b), both $\mathrm{D}$ and $\mathrm{A}_{\text {eff }}$ are decreasing by a factor of $\sim 10$ from the top left corner of the map to a point close to $\mathrm{D}=0$. Fig. 2(a) shows the simulated propagation of a $400 \mathrm{fs}$ soliton (dispersion length $\sim 5 \mathrm{~m}$ ) at fiber input through $50 \mathrm{~m}$ of low-loss holey fiber with the corresponding profiles for $\mathrm{D}$ and $\mathrm{A}_{\text {eff. }}$ After $\sim 20 \mathrm{~m}$ of fiber, the observed width deviates from the analytic expression (1). A closer analysis reveals that two effects prevent further compression at this point: (i) As the pulse broadens spectrally and the fiber parameters change, a zero-dispersion wavelength is found close to the soliton wavelength towards the fiber end and thus the soliton starts to shed energy into dispersive waves [4]. (ii) The large decrease in $\mathrm{A}_{\text {eff }}$ (increase of nonlinearity) leads to temporal broadening by Raman soliton self-frequency shifting (SSFS). In order to avoid these limiting factors, holey fiber parameters have to be chosen in the top left area of the map, Fig, 1(a), with an endpoint near the line of zero dispersion slope Ds=0.

Path 2 of Fig. 1(b) represents such a choice. Here, D decreases by a factor of $\sim 5$ and $\mathrm{A}_{\text {eff }}$ by a factor of $\sim 2.5$ along the fiber. The corresponding simulation results, Fig. 2(b), closely follow the analytic approximation (1), which suggests that the soliton compression is indeed adiabatic. A small SSFS is still observed in the corresponding spectrum but no dispersive waves are generated. A $400 \mathrm{fs}$ soliton pulse is compressed down to $33 \mathrm{fs}$, a compression factor of $\sim 12$. The adiabaticity of the soliton compression is further confirmed by the fact that simulations using paths with the same end points as Path 2 but alternative routes in between yield very similar performances.

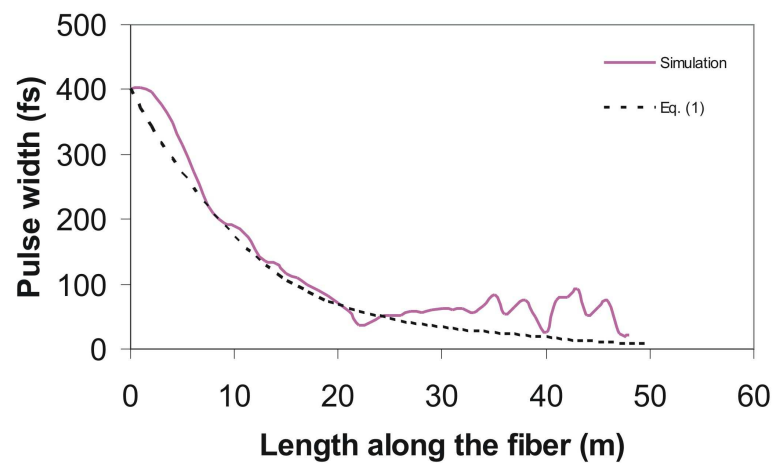

(a)

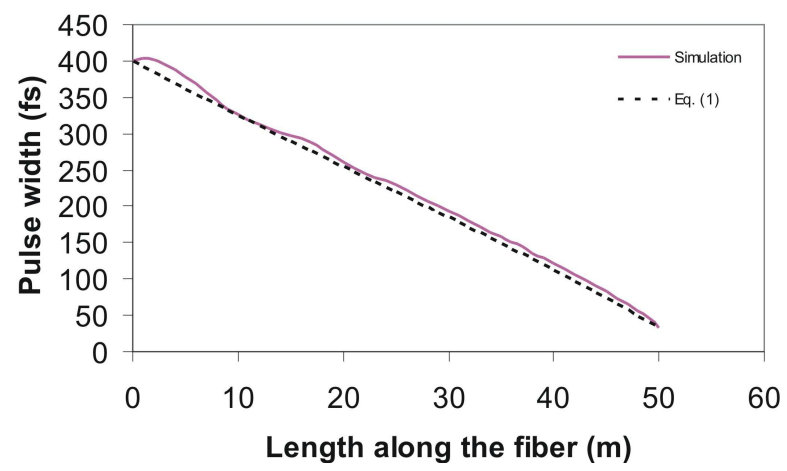

(b)

Fig. 2. Simulated pulse widths (FWHM) in fibers with dispersion and effective area profiles along (a) Path 1 and (b) Path 2 of Fig. 1 . In both cases the fiber parameters decrease with a constant rate of variation along the $50 \mathrm{~m}$ length of fiber.

\section{Conclusions}

We have investigated adiabatic compression of femtosecond solitons in silica holey fibers of decreasing dispersion and effective mode area. These parameters are directly related to the structural design parameters $\Lambda$ and $d / \Lambda$. A compression factor of 12 has been obtained for low-loss fibers in the adiabatic regime. Minimizing the fiber length required for adiabatic compression and nonadiabatic compression in much shorter fibers is currently under investigation.

\section{References}

[1] S. V. Chernikov, E. M. Dianov, D. J. Richardson and D. N. Payne, "Soliton pulse compression in dispersion-decreasing fiber" Opt. Lett. 18, 476 (1993).

[2] J. Hu, B. S. Marks, C. R. Menyuk, J. Kim, T. F. Carruthers, B. M. Wright, T. F. Taunay, and E. J. Friebele, "Pulse compression using a tapered microstructure optical fiber," Opt. Express 14, 4026 (2006).

[3] M. L. V. Tse, P. Horak, J. H. V. Price, F. Poletti, F. He, and D. J. Richardson, "Pulse compression at $1.06 \mu \mathrm{m}$ in dispersion-decreasing holey fibers," Opt. Lett. 31, 3504 (2006).

[4] A. V. Husakou and J. Herrmann, "Supercontinuum generation of higher-order solitons by fission in photonic crystal fibers," Phys. Rev. Lett. 87, 203901 (2001). 